\title{
Adaptation d'une méthode colorimétrique à la mesure automatisée de l'activité cataly- tique des cholinestérases dans un plasma et dans un hémolysat de sang total
}

\section{Adaptation of a colorimetric method for auto- mated measurement of cholinesterase activity in plasma and whole blood hemolysate}

Denis CHIANÉA*(1) ${ }^{* a b i e n n e ~}$ ADAM $^{(1)}$, Jean-Louis CAPRON ${ }^{(2)}$, Hubert CORBÉ(1)

(1) Laboratoire de Biochimie, Toxicologie et Pharmacologie Cliniques, H.I.A. Clermont-Tonnerre, rue Colonel Fonferrier - BP 41 - 29240 BREST Naval (2) Service de Médecine de Prévention, D.C.N. - 29240 BREST Naval

*Auteur à qui adresser la correspondance : Le Pharmacien Chimiste Denis CHIANÉA, Laboratoire de Biochimie, Toxicologie et Pharmacologie Cliniques, Hôpital d'Instruction des Armées Clermont-Tonnerre, rue Colonel Fonferrier - BP 41 - 29240 BREST Naval - Tél : 0298437228 - Fax : 0298437457

(Reçu le 18 octobre 2000 ; accepté le 20 décembre 2000)

\section{RÉSUMÉ}

La mesure de l'activité des cholinestérases dans le plasma permet le diagnostic d'une intoxication aiguë ou chronique par un inhibiteur des chlolinestérases. D'après la littérature, l'activité intra-érythrocytaire est un marqueur pertinent dans les intoxications chroniques et constitue un meilleur indicateur des effets biologiques des inhibiteurs des cholinestérases que l'activité plasmatique. Les auteurs proposent une adaptation de la méthode colorimétrique manuelle en mode biréactif Cholinestérase MPR2 Roche. La technique proposée permet la mesure automatisée à $37^{\circ} \mathrm{C}$ de l'activité des cholinestérases dans un plasma ou dans un hémolysat de sang total sur analyseur multiparamétrique Cobas ${ }^{\circledR}$ Mira ABX Diagnostics ; l'activité intra-érythrocytaire est obtenue par calcul à partir des deux activités précédentes et de l'hématocrite. La technique est linéaire entre 19 et 959 U/L. Trente sérums et trente hémolysats de sang total ont été analysés avec la technique proposée et avec une méthode de référence en mode mono-réactif : Cholinestérase [PTC] Sigma Diagnostics. La comparaison des résultats rend compte d'un

\section{SUMMARY}

The measurement of plasma cholinesterase activity is used to diagnose acute or chronic poisoning with cholinesterase inhibitors. According to the literature, the erythrocyte cholinesterase activity is a better biomarker for chronic cholinesterase inhibitor poisoning than plasma cholinesterase activity. The authors propose an adaptation of the colorimetric method in bi-reagent form Cholinesterase MPR2 Roche. The proposed technique allows automated measurement at $37^{\circ} \mathrm{C}$ of the cholinesterase activity in plasma or hemolysate of whole blood on a Cobas ${ }^{\circledR}$ Mira ABX Diagnostics chemistry system; erythrocyte cholinesterase activity is obtained by calculation from the previous two activities and from the hematocrit value. The technique is linear between 19 and 959 U/L. Thirty sera and thirty hemolysates of whole blood were analysed with the proposed technique and with a reference method in mono-reagent form : Cholinesterase [PTC] Sigma Diagnostics. The comparison of results yields a correlation coefficient of 0,99 for plasma and 0,91 for whole blood hemolysate. The automation reduces imprecision due 
facteur de corrélation égal à 0,99 pour les échantillons plasmatiques et à 0,91 pour les hémolysats de sang total. L'automatisation réduit les imprécisions liées à l'utilisation de petits volumes de prise d'essai $(4 \mu \mathrm{l})$ et de réactif déclenchant $(28 \mu l)$. Elle permet de rendre un résultat dans un délai de cinq minutes après traitement des échantillons biologiques. L'emploi d'un bi-réactif est économiquement rentable particulièrement pour l'analyse ponctuelle de petites séries d'échantillons biologiques: les réactifs sont stables pendant six semaines à $+4{ }^{\circ} \mathrm{C}$ et à l'abri de la lumière après reconstitution.

\section{MOTS-CLÉS}

Acétylcholinestérase intraérythrocytaire, carbamates, intoxication, méthode colorimétrique, organophosphorés, pesticides.

\section{Introduction}

Les cholinestérases forment un système enzymatique complexe composé de nombreuses isoformes capables de cliver les esters de la choline. On distingue l'acétylcholinestérase, présente dans le tissu nerveux ou les érythrocytes, et la butyrylcholinestérase (anciennement appelée pseudo-cholinestérase) majoritaire dans le plasma. L'acétylcholinestérase régule l'influx nerveux dans les synapses entre les neurones et les muscles en hydrolysant rapidement l'acétylcholine. Le rôle physiologique de la butyrylcholinestérase n'est pas exactement connu (1). L'inhibition des cholinestérases provoque un syndrome cholinergique par accumulation de l'acétylcholine au niveau des récepteurs cholinergiques muscariniques et nicotiniques des terminaisons nerveuses ; la symptomatologie dépend de la dose et de la voie de pénétration du toxique (2). Les principaux inhibiteurs des cholinestérases sont les organophosphorés, les carbamates et les organosulfonés (1).

La mesure de l'activité catalytique des cholinestérases dans le sérum et les érythrocytes présente un intérêt dans les intoxications aiguës ou chroniques par les inhibiteurs des cholinestérases.

Les auteurs proposent l'adaptation d'une technique colorimétrique permettant la mesure automatisée à $37^{\circ} \mathrm{C}$ de l'activité des cholinestérases dans un plasma et dans un hémolysat de sang total ; l'activité intra-érythrocytaire peut être déduite par calcul à partir des deux paramètres précédents.

\section{Matériel et méthode}

\section{Principe :}

Dans l'adaptation proposée, les cholinestérases contenues dans un plasma ou un hémolysat de sang total hydrolysent le substrat acétylthiocholine en milieu to manipulation of small sample volume $(4 \mu \mathrm{l})$ and start-reagent $(28 \mu \mathrm{l})$. The proposed technique allows to obtain a result in five minutes after treatment of the samples. The use of bi-reagent is economical particularly for the analysis of a small series of samples : after reconstruction, the reagent solutions are stable during six weeks at $+4{ }^{\circ} \mathrm{C}$ if shielded from the light.

\section{KEY-WORDS}

Carbamates, colorimetric method, erythrocyte acetylcholinesterase, organophosphates, pesticides, poisoning.

thermostaté à $37^{\circ} \mathrm{C}$, pour former de la thiocholine. La thiocholine réagit avec un chromogène (le dithio-bis(nitrobenzoate)) et l'élévation moyenne des densités optiques est mesurée à $405 \mathrm{~nm}$ sur analyseur Cobas $^{\circledR}$ Mira (ABX Diagnostics). Les activités catalytiques des échantillons plasmatiques et des hémolysats sont obtenues à partir d'un droite d'étalonnage en deux points commune aux deux milieux biologiques. L'activité enzymatique intra-érythrocytaire est obtenue par calcul, en déduisant l'activité plasmatique de l'activité de l'hémolysat (activité totale), rapportée à la valeur de l'hématocrite.

Les réactifs utilisés sont présentés sous forme de biréactifs : coffret Cholinestérase $M P R 2$, référence 124117*, substrat acétylthiocholine des laboratoires Roche. Ce coffret est initialement destiné à la mesure manuelle à $25^{\circ} \mathrm{C}$ des cholinestérases dans un plasma ou un sérum (3).

\section{Méthode de référence :}

Cette adaptation a été validée (4) avec une technique automatisée sur analyseur multiparamétrique Cobas $^{(}$ Mira : coffret "Cholinestérase [PTC]", référence 42210, des laboratoires Sigma Diagnostics (5). Le coffret est composé d'un mono-réactif (substrat propionylthiocholine et tampon-chromogène en mélange). La méthode permet la mesure quantitative de l'activité des cholinestérases dans un plasma et dans un hémolysat de sang total.

\section{Prélèvements et traitement des échan- tillons biologiques :}

L'étude est conduite de façon anonyme sur trente adultes, sans distinction d'âge ou de sexe. Les échantillons biologiques sont obtenus dans le cadre de bilans d'aptitude, à partir de prélèvements réalisés par ponction veineuse au pli du coude. Ces bilans d'aptitudes 
nécessitent un prélèvement sur tube hépariné pour les analyses biochimiques, un prélèvement sur tube sec pour les analyses sérologiques et un prélèvement sur EDTA pour la numération-formule sanguine. Après réalisation de la numération-formule et saisie de l'hématocrite, le tube EDTA est récupéré pour la mesure des cholinestérases dans le plasma et dans l'hémolysat de sang total.

\section{Échantillons d'hémolysat de sang total :}

Un hémolysat est préparé en mélangeant 100 microlitres de sang total, préalablement homogénéisé, avec 1900 microlitres d'eau distillée (ce qui correspond à une dilution du sang total au vingtième). Le mélange est homogénéisé sur un mélangeur rotatif Vortex ${ }^{\circledR}$ pendant dix secondes. L'hémolyse est renforcée en plaçant la suspension à $+4{ }^{\circ} \mathrm{C}$ pendant quinze minutes.

L'hémolysat est centrifugé à trois mille tours par minute pendant dix minutes, puis le surnageant est récupéré pour la mesure des activités catalytiques.

L'hémolyse doit être réalisée dans les trente minutes pour limiter l'influence du métabolisme cellulaire sur les cholinestérases (6). Les hémolysats peuvent être conservés entre +2 et $+6^{\circ} \mathrm{C}$, mais leur activité catalytique doit être mesurée dans les huit heures (5).

\section{Échantillons plasmatiques :}

Les échantillons sont centrifugés à trois mille tours par minute pendant quinze minutes. La séparation du plasma du culot de globules rouges doit être effectuée dans les trente minutes pour limiter l'influence du métabolisme cellulaire (6). Le plasma est dilué au sixième dans une solution physiologique de chlorure de sodium avant analyse. Les plasmas présentant une hémolyse visible à l'œil nu sont rejetés. L'activité des cholinestérases est stable dans un sérum non dilué pendant deux semaines lorsque le sérum est conservé entre +2 et $+6^{\circ} \mathrm{C}$, et pendant trois mois à $-20^{\circ} \mathrm{C}(7)$. La présence d'EDTA n'inhibe pas l'activité des cholinestérases.

\section{Standards et contrôles :}

Le standard utilisé pour l'étalonnage linéaire est : Calibrator (human) Rocal, réf. 2052 601, des laboratoires Roche. Le contrôle de qualité interne utilisé est : Control Serum N (human), réf. 2052 547, des laboratoires Roche. Le contrôle Precinorm ${ }^{\circledR} U$, référence 171735, Roche est utilisé pour l'étude de reproductibilité.

Les valeurs cibles précisées en U/L sur les notices d'emploi du standard et des contrôles sont données pour une technique à la butyrylthiocholine; cette valeur doit être multipliée par un facteur de conversion global de 0,3512 pour l'acétylthiocholine. Ce facteur a été déterminé statistiquement à partir de trente mesures manuelles du standard Calibrator (human) Rocal Roche réalisées à $25^{\circ} \mathrm{C}$ puis à $37^{\circ} \mathrm{C}$ sur spectrophotomètre Cary 1 Varian thermostaté, avec les volumes d'échantillon et de réactifs recommandés par le fabriquant du coffret de réactifs :

Calcul du facteur de conversion de température $\left(\mathrm{FT}^{\circ}\right)$ : $F T^{\circ}=\frac{\text { moyenne des activités mesurées à } 37^{\circ} \mathrm{C}}{\text { moyenne des activités mesurées à } 25^{\circ} \mathrm{C}}$

Soit, $\mathrm{FT}^{\circ}=1,86$.

Calcul du facteur de conversion global (FG) :

$F G=F T^{\circ} \times \frac{\text { moyenne des activités mesurées à } 25^{\circ} \mathrm{C}}{\text { valeur théorique pour }}$

Conditions opératoires et programmation

de l'automate (figure 1) :

- mode de calibration : étalonnage linéaire en deux points avec dilutions automatiques du standard Calibrator (human) Rocal Roche au demi et au cinquième ; le contrôle Control Serum N (human) Roche est dilué manuellement au sixième dans de l'eau distillée avant analyse.

- chaque point de gamme est mesuré en double, avec exclusion de l'étalonnage si la déviation moyenne est supérieure à $6 \%$;

- mode de mesure : absorbance ;

- mode de calcul de l'absorbance : cinétique ;

- sens de la réaction : croissant ;

- mode réactionnel : D-R-S-SR1 (dilution et biréactif) ;

- intervalle de calibration : chaque jour ;

- longueur d'onde de mesure : $405 \mathrm{~nm}$;

- unité de mesure : U/L ;

- température de réaction $37^{\circ} \mathrm{C}$;

- blanc-réactif automatique ;

- lavage automatique de la pointe réactif avant le pipetage du réactif de travail ;

- prise d'essai de l'échantillon plasmatique : 4,0 microlitres ;

- volume de dilution de l'échantillon dans de l'eau distillée : 8,0 microlitres ;

- volume de tampon/chromogène : 400 microlitres ;

- volume de réactif déclenchant (substrat acétylthiocholine) : 28,0 microlitres ;

- facteur de conversion interne : néant ;

- cycle de lecture : premier point de mésure à 150 secondes (first : 6), dernier point de mesure à 250 secondes (last : 10) ; 


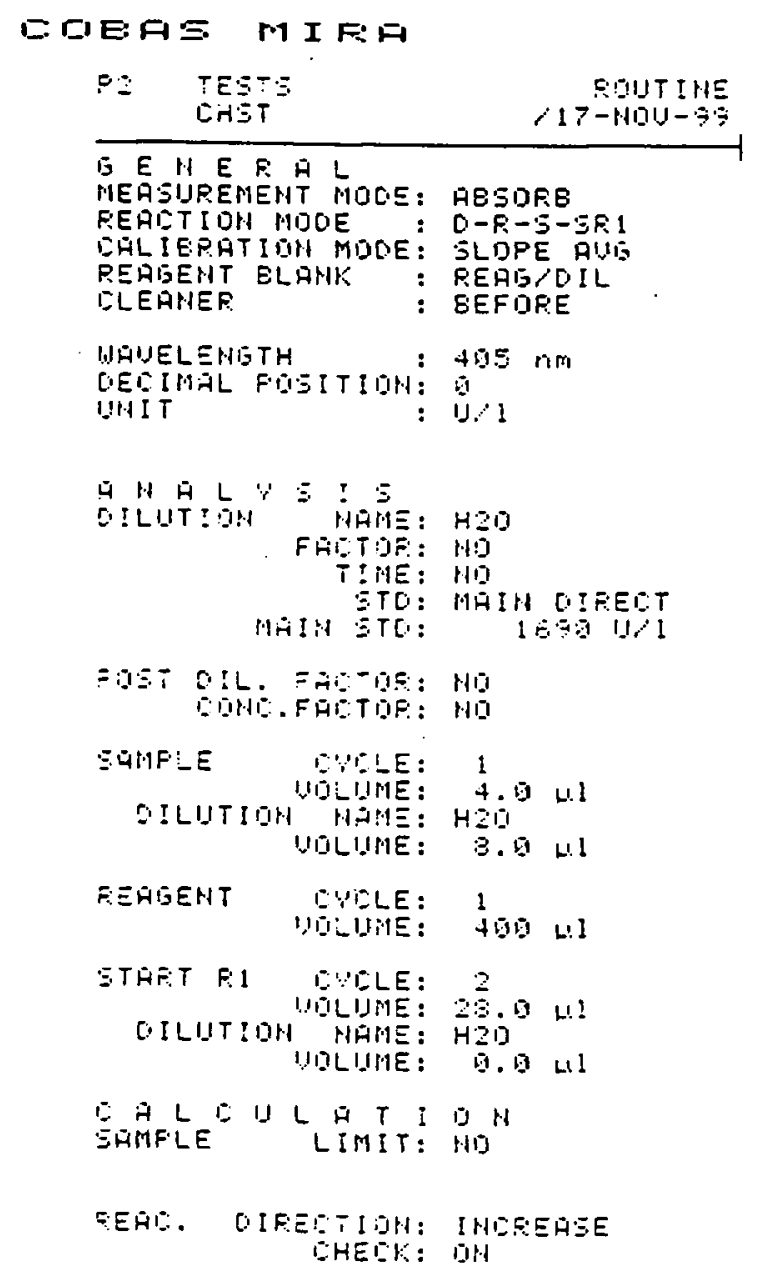

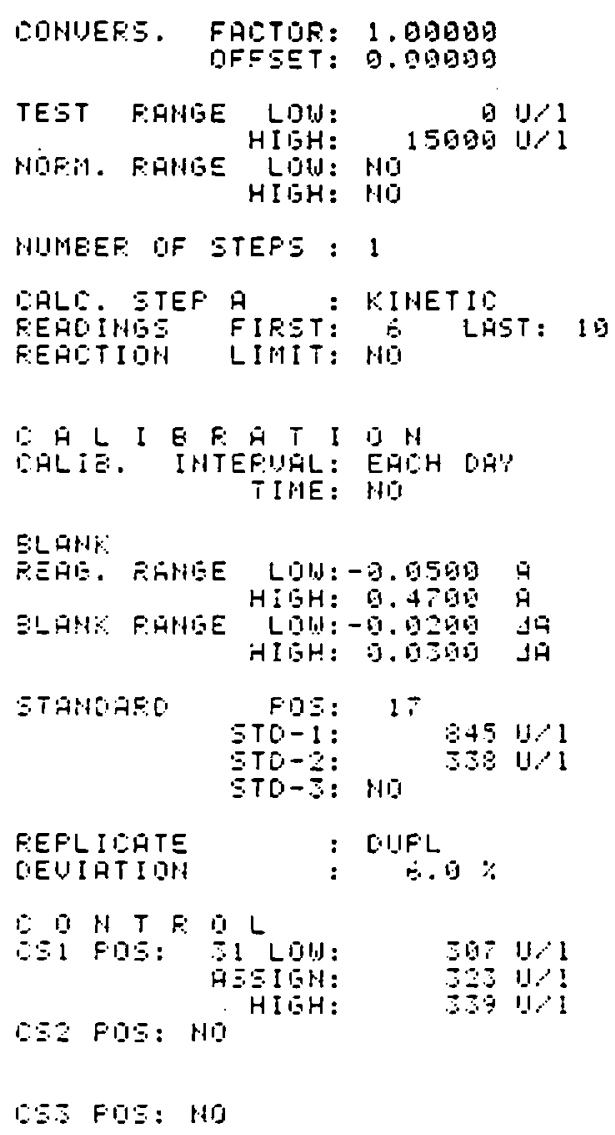

$21-A F R-B D$
Figure 1 : Programmation de l'analyseur Cobas Mira ${ }^{\circledR}$ pour la mesure de l'activité des cholinestérases dans un plasma ou dans un hémolysat de sang total.

\section{Calcul et expression des résultats :}

Les unités utilisées sont les unités internationales : U/L.

L'activité plasmatique (PChE) correspond à l'activité mesurée sur plasma prédilué au sixième $(\mathrm{PdChE})$ multipliée par un facteur 6 :

$$
P C h E=6 \times P d C h E
$$

L'activité totale (TChE) correspond à l'activité mesurée dans l'hémolysat $(\mathrm{HChE})$ multipliée par un facteur 20 (correspondant à la dilution du sang total dans l'eau distillée) :

$$
T C h E=20 \times H C h E
$$

L'activité intraérythrocytaire (EChE) est obtenue par calcul à partir des deux activités précédentes, en tenant compte du résultat de l'hématocrite $(\mathrm{Ht})$ exprimée en valeurs décimales :

$$
E C h E=\frac{[T C h E-(P C h E x(1-H t))]}{H t}
$$

\section{Résultats}

\section{Linéarité :}

L'étude de linéarité repose sur la mesure par série, du contrôle Control Serum $N$ (human) Roche pur et de quatre dilutions de ce contrôle $(1 / 2,1 / 10,1 / 50$ et $1 / 100)$; chaque point de gamme est analysé dans trois séries différentes (figure 2).

L'équation de régression linéaire est : $\mathrm{y}=0,9632 \mathrm{x}+$
5,901 avec r2 =0,9995. Le domaine de linéarité s'étend de $19 \mathrm{U} / \mathrm{L}$ à $959 \mathrm{U} / \mathrm{L}$.

\section{Précision :}

\section{Répétabilité :}

La répétabilité est mesurée en une seule série sur dix fractions aliquotes du calibrateur Calibrator (human) Rocal Roche dilué au 1/6. Le coefficient de variation est de $1,38 \%$ (tableau I).

\section{Tableau I : Étude de Répétabilité.}

\begin{tabular}{|c|c|}
\hline répétabilité & $\begin{array}{c}\text { Calibrateur Rocal Roche dilué au 1/6 } \\
\text { (valeur théorique : 320 U/1) }\end{array}$ \\
\hline & 307 \\
& 307 \\
& 308 \\
mesures & 304 \\
intrasérie & 308 \\
& 307 \\
& 311 \\
& 302 \\
& 298 \\
& 299 \\
\hline moyenne (U/L) & $\mathbf{3 0 5 , 1}$ \\
écart type (U/L) & $\mathbf{4 , 2 3}$ \\
CV (\%) & $\mathbf{1 , 3 8}$ \\
\hline
\end{tabular}


Annales de Toxicologie Analytique, vol. XIII, nº 1,2001

Tableau II : Étude de reproductibilité

\begin{tabular}{|c|c|c|}
\hline reproductibilité & $\begin{array}{c}\text { Contrôle Serum N Roche dilué au 1/6 } \\
\text { (valeur cible théorique : 320 U/L) }\end{array}$ & $\begin{array}{c}\text { Contrôle Precinorm U Roche dilué au 1/6 } \\
\text { (valeur cible théorique : 428 U/L) }\end{array}$ \\
\hline J1 & 325 & 426 \\
J2 & 327 & 411 \\
J3 & 326 & 433 \\
J4 & 332 & 428 \\
J5 & 322 & 417 \\
J6 & 325 & 419 \\
J7 & 332 & 429 \\
J8 & 305 & 385 \\
J9 & 326 & 427 \\
J10 & 323 & 426 \\
\hline moyenne (U/L) & $\mathbf{3 2 4 , 3}$ & $\mathbf{4 2 0}$ \\
écart type (U/L) & $\mathbf{7 , 5 4}$ & $\mathbf{1 3 , 9 5}$ \\
CV (\%) & $\mathbf{2 , 3 3}$ & $\mathbf{3 , 3 2}$ \\
\hline
\end{tabular}

\section{Reproductibilité :}

L'essai consiste à analyser pendant dix jours deux niveaux de contrôle (tableau II) : une dilution au 1/6 du contrôle Control Serum N (human) Roche (contrôle bas) et une dilution au 1/6 du contrôle Precinorm ${ }^{\circledR} U$ Roche (contrôle haut). Le coefficient de variation est égale à $2,33 \%$ pour le contrôle bas et à $3,32 \%$ pour le contrôle haut.

\section{Sensibilité :}

La sensibilité est définie comme étant la plus petite concentration mesurable pouvant être distinguée de zéro, avec un intervalle de confiance de $95 \%$. Elle est évaluée sur dix échantillons de Control Serum $N$ (human) Roche dilués au centième et est égale à 19 $\mathrm{U} / \mathrm{L}$, avec un coefficient de variation de $4,35 \%$.

\section{Évaluation de l'exactitude par corrélation avec une technique de référence :}

Les activités des cholinestérases mesurées avec l'adaptation technique proposée sur 30 échantillons plasmatiques humains et sur 30 hémolysats humains sont comparées à celles qui sont obtenues avec la méthode de référence (figures 3 et 4 ). Il existe une bonne corrélation entre les deux techniques pour les deux milieux biologiques :

- mesures plasmatiques : le facteur de corrélation est $\mathrm{r}^{2}=0,99$ et l'équation de la droite de régression est $\mathrm{y}=$ $0,5744 . x+65,652$;

- mesures dans les hémolysats de sang total : le facteur de corrélation est $\mathrm{r}^{2}=0,91$ et l'équation de la droite de régression est $\mathrm{y}=0,6876 . \mathrm{x}-84,978$.

La méthode statistique des couples comparant les moyennes de deux séries de faible effectif (8) est utilisée pour évaluer la moyenne des différences des mesures effectuées avec les deux techniques pour chaque échantillon de plasma ou d'hémolysat. Pour les deux types de milieu biologique, les moyennes des mesures trouvées avec les deux techniques ne diffèrent pas significativement, comme le montrent les résultats suivants :

- pour les échantillons plasmatiques : la moyenne des différences est égale à 1,899 et $/ t /=0,92$. Cette valeur est inférieure au seuil donné par la table de t pour un nombre de degrés de liberté $(\mathrm{n}-1)=29$ et un risque $\alpha=$ $5 \%$, soit 2,045 .

- pour les hémolysats : la moyenne des différences est égale à $-0,79$ et $/ t /=0,23$. Cette valeur est inférieure au seuil donné par la table de t pour un nombre de degrés de liberté $(n-1)=29$ et un risque $\alpha=5 \%$, soit 2,045 .

\section{Valeurs de référence :}

Elles sont calculées à partir des valeurs statistiques obtenues lors de la corrélation intertechniques pour chaque milieu biologique : moyenne \pm 2 écarts types.

Activité cholinestérasique dans le plasma :

2377 - $5845 \mathrm{U} / \mathrm{L}$.

Activité cholinestérasique dans le sang total :

$4791-8315 \mathrm{U} / \mathrm{L}$. 

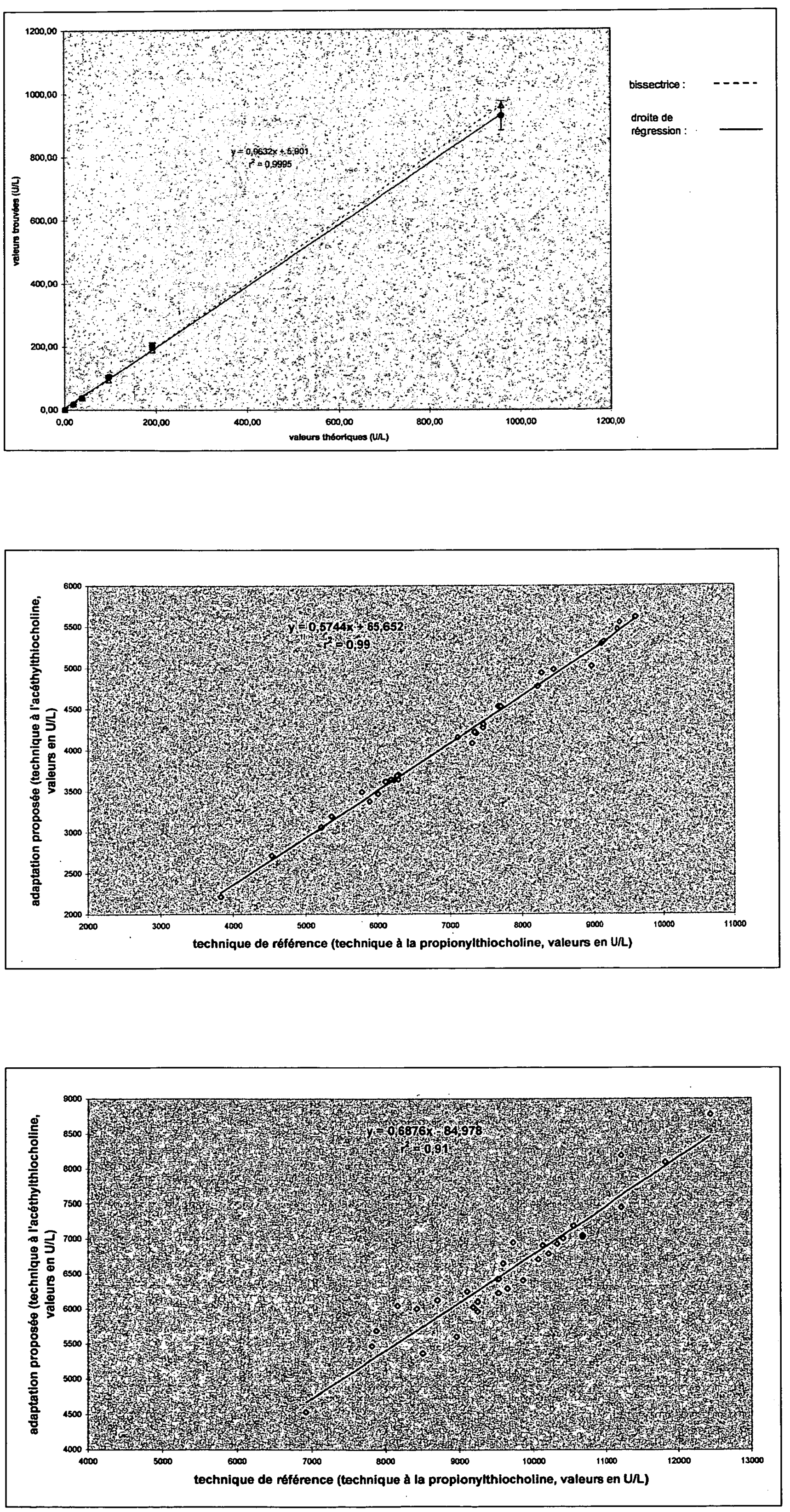

Figure 2 : Étude de linéarité.

Figure 3 : Mesure de l'activité des cholinestérases dans les plasmas, corrélation avec la technique de référence.

Figure 4 : Mesure de l'activité des cholinestérases dans les hémolysats de sang total, corrélation avec la technique de référence. 
Activité cholinestérasique érythrocytaire : 6830 - $12062 \mathrm{U} / \mathrm{L}$.

Le nombre de sujets sur lesquels ont été établies les valeurs de référence est limité et ces résultats devront être complétés par des études plus approfondies sur des populations plus importantes, en tenant compte de l'âge, du sexe et de l'état physiopathologique des patients étudiés. Pour le plasma, l'intervalle de référence calculé est significativement différent des taux usuels plasmatiques figurant dans la notice technique du coffret Cholinestérase MPR2 Roche (3) établis d'après les travaux de Weber $\mathrm{H}$. : en effet après correction par le facteur de conversion de température théorique calculé plus haut $\left(F T^{\circ}=1,86\right)$ les taux usuels ramenés à $37^{\circ} \mathrm{C}$ sont compris entre 3534 et $7068 \mathrm{U} / \mathrm{L}$. Ce décalage peut s'expliquer par le calcul des activités enzymatique des échantillons à partir d'un sérum de calibration. Les facteurs de corrections utilisés dans la technique proposée (facteur de conversion de température et facteur de conversion global) ont été calculés à partir d'un sérum de calibration humain qui ne présente pas nécessairement le même profil isoenzymatique que celui de tous les patients témoins ayant servis à établir les valeurs de référence. Dans ces conditions, une simple extrapolation des taux usuels à partir d'une formule de calcul est délicate et le recours à une population témoin pour établir les valeurs de référence s'avère indispensable.

\section{Discussion}

\section{Critères de praticabilité de la méthode :}

Chez l'individu sain, l'intensité du signal obtenu lors de la mesure des activités cholinestérasiques plasmatiques par méthode colorimétrique à $25^{\circ} \mathrm{C}$ et à fortiori à $37{ }^{\circ} \mathrm{C}$, impose des prédilutions importantes des échantillons biologiques ou l'utilisation de petits volumes de prise d'essai souvent à l'origine d'imprécision dans les méthodes manuelles. L'automatisation de ce type de méthode limite au maximum ces imprécisions et permet de rendre un résultat en cinq minutes, après traitement des échantillons biologiques.

La forme bi-réactif (tampon-chromogène et substrat acétylthiocholine) du coffret Roche présente l'avantage d'être stable pendant six semaines après reconstitution si les deux réactifs sont conservés en flacon opaque à $+4{ }^{\circ} \mathrm{C}$; les formes monoréactifs commercialisées offrent généralement des durées de stabilité beaucoup plus restreintes après reconstitution : quatre heures à température ambiante ou un jour à $+4^{\circ} \mathrm{C}$ pour le monoréactif Cholinestérase [PTC] Sigma Diagnostics. La durée de stabilité des réactifs du coffret utilisé dans notre adaptation technique est un avantage économique pour de petites séries d'analyses.

\section{Facteurs de variation biologiques :}

Intoxications aiguës et chroniques aux inhibiteurs des cholinestérases :

En France, dans le cadre de la surveillance médicale des salariés, l'arrêté du 11 juillet 1977 prévoit une surveillance médicale spéciale pour des travailleurs exposés aux esters de l'acide phosphorique (9). Il est de bonne pratique dans ce cadre de mesurer périodiquement l'activité des cholinestérases sériques ou érythrocytaires, mais aucune obligation n'existe en la matière. Les armées, quant à elles, imposent une surveillance médicale régulière des personnels manipulant des insecticides organophosphorés ou dérivés des carbamates. Cette surveillance médicale comporte la réalisation semestrielle d'un examen clinique complet, d'un bilan hématologique et d'une mesure quantitative de l'activité des cholinestérases sériques. Une atteinte organique sérieuse, quelle qu'en soit l'origine, ou la baisse de $25 \%$ de l'activité des cholinestérases sériques impose le retrait du personnel de son poste de travail jusqu'à guérison ou rétablissement d'une activité enzymatique satisfaisante (10). L'activité sérique diminue plus précocement et redevient normale plus rapidement que l'activité globulaire (11). Plusieurs travaux montrent l'intérêt de la mesure de l'activité des cholinestérases intraérythrocytaires dans le diagnostic et le suivi des intoxications chroniques $(12,13,14)$.

Pour certains auteurs, le délai de retour à une activité enzymatique érythrocytaire normale après inhibition irréversible d'une partie des cholinestérases, est compris entre quatre et six mois (15). Lors d'une intoxication grave, il est admis que les enzymes ne sont plus réactivables si l'activité sérique des cholinestérases reste effondrée après 24 heures de traitement à la pralidoxime (2).

\section{Autres facteurs de variation biologiques :}

Certaines affections non liées à une intoxication peuvent être à l'origine d'une diminution non spécifique de l'activité totale des cholinestérases (plasmatique et globulaire) : déficits congénitaux, hépatite virale, cirrhose du foie, hépatocarcinome, insuffisance hépatocellulaire, anémie, dermato-myosite, syndrome neuromusculaire ou encore malnutrition. L'activité intraérythrocytaire est abaissée dans l'hémoglobinurie nocturne paroxystique ou maladie de Marchiafava-Michelli (11).

Une augmentation de l'activité catalytique totale peut être observée dans le syndrome néphrotique, l'obésité ou l'infarctus du myocarde (11). 


\section{Conclusion}

La mesure de l'activité catalytique des cholinestérases dans le plasma ou le sérum est essentielle pour le diagnostic d'une intoxication aiguë et chronique par un inhibiteur des cholinestérases. Néanmoins, d'après la littérature, la détermination de l'activité intra-érythrocytaire permet de mieux préjuger de l'importance du déficit enzymatique, notamment au cours des intoxications chroniques. L'adaptation technique proposée présente l'avantage de pouvoir évaluer rapidement l'activité des cholinestérases par mesure cinétique à $37^{\circ} \mathrm{C}$ sur analyseur multiparamétrique Cobas ${ }^{\circledR}$ Mira. Les mesures peuvent être effectuées aussi bien dans le plasma que dans des hémolysats de sang total et permettent de calculer l'activité intra-érythrocytaire. Le mode biréactif choisi présente l'avantage de pouvoir utiliser des solutions de travail stables pendant six semaines après reconstitution si elles sont conservées à $+4^{\circ} \mathrm{C}$ en flacon opaque ; cette durée de stabilité est bien adaptée à l'analyse ponctuelle de petites séries d'échantillons biologiques.

\section{Références}

1. Curtil C., Masson P. Le vieillissement des cholinestérases après inhibition par les organophosphorés, Ann. pharmaceutiques françaises, 1993 ; 51 (2), 63-77.

2. Blanchet J.M., Noto R., Pailler F.M., Renaudeau C., Ricordel I. Les agressions chimiques, France-Sélection, Paris, 1997 ; 15-18.

3. Notice technique, Cholinestérase substrat acétylthiocholine MPR2 réf. 124117, Roche, août 1999.

4. Vassault A., Grafmeyer D., Naudin C., Dumont G., Bailly M., Henny J., Gerhardt M.F., Georges P. Protocole de validation des techniques, Commission Validation de Techniques, I.S.B., $1985 ; 11$ (1), 5-45.

5. Notice technique, Cholinesterase (PTC) Application for Cobas ${ }^{\circledR}$ Mira Chemistry Systems, Procedure $\mathrm{N}^{\circ} 422$, Sigma Diagnostics, revision 25/08/1995.

6. Desch G., Duchassaing D., Eloy O., Harzic M., Ghnassia J.C., Linget C., Meillet D., Piemont Y., Szymanowicz A., Terré C., Thoinet S., Troalen F. Échantillons biologiques - Phase préanalytique et prélèvements en biologie médicale, collection Option Bio, Editors Elsevier, Nancy, $1998 ; 143$.

7. Henry R.J., Cannon D.C., Winkelman J.W. Clinical Chemistry - Principles and Techniques, 2nd ed., Editors Harper and Row, Hagerstown [MD], 1974 ; 919.

8. Schwartz D. Méthodes statistiques à l'usage des médecins et des biologistes, 4th ed., Médecine-Sciences Flammarion, Paris, 1996 ; 151-162.

9. Arrêté du 11 juillet 1977 fixant la liste des travaux nécessitant une surveillance médicale spéciale, J.O., 24 juillet 1977.

10.I.M. $\mathrm{n}^{\circ}$ 710/DEF/DCSSA/AST/TEC sur la désinsectisation et la dératisation dans les armées, du 12 mars 1997 (BOC/PP, 12 mai 1997, $\mathrm{N}^{\circ}$ 20, 2229 - 2264).

11.Funes A. Guide des analyses spécialisées, 2nd ed., Laboratoire CERBA, Cergy Pontoise, 1989 ; 146-147.

12. Walker C.H. Biochemical responses as indicators of toxic effects of chemicals in ecosystem, Toxicol. Let., 1992 ; 64, 527-533.

13.Meuling W.J.A., Jongen M.J.M., van Hemmen J.J. An automated method for the determination of acetyl and pseudo cholinesterase in hemolysed whole blood, Am. J. Ind. Med., 1992 ; 22, 231-241.

14.Mc Connell R., Magnotti R. Screening for insecticide over-exposure under field conditions - a re-evaluation of the titrimetric cholinesterase kit, Am. J. Public Health, $1994 ; 84,479-481$.

15.Gomes J., Lloyd O., Revitt D.M., Norman J.N. Erythrocyte cholinesterase activity levels in desert farm workers, Occup. Med., 1997 ; 47, 90-94. 